\title{
Hybrid recursive regularized lattice Boltzmann simulation of humid air with application to meteorological flows
}

\author{
Yongliang Feng,* Pierre Boivin, Jérôme Jacob, and Pierre Sagaut ${ }^{\dagger}$ \\ Aix Marseille Univ, CNRS, \\ Centrale Marseille, M2P2, Marseille, France
}

(Dated: July 18, 2019)

\begin{abstract}
An extended version of the hybrid recursive regularized Lattice-Boltzmann model which incorporates external force is developed to simulate humid air flows with phase change mechanisms under the Boussinesq approximation. Mass and momentum conservation equations are solved by a regularized lattice Boltzmann approach well suited for high Reynolds number flows, whereas the energy and humidity related equations are solved by a finite volume approach. Two options are investigated to account for cloud formation in atmospheric flow simulations. The first option considers a single conservation equation for total water and an appropriate invariant variable of temperature. In the other approach, liquid and vapor are considered via two separated equations, and phase transition is accounted for via a relaxation procedure. The obtained models are then systematically validated on four well-established benchmark problems including a double diffusive Rayleigh Bénard convection of humid air, 2D and 3D thermal moist rising bubble under convective atmospheric environment as well as a shallow cumulus convection in framework of large-eddy simulation.

PACS numbers: 47.55.t, 47.11.j,47.45.-n

Keywords: lattice Boltzmann method; atmospheric convection; cloud dynamics; shallow cumulus convection
\end{abstract}

\section{INTRODUCTION}

Due to its central role in weather forecast [1] for being a key element in cloud formation and rain, atmospheric moisture has a significant impact on air quality and consequently on public health [2]. Moist atmospheric dynamics is composed of motions occurring on a wide range of temporal and spatial scales, from $0.1-1 \mathrm{~km}$ for the convective macro-structures (e.g. clouds), down to the mm-sized water drop governing the atmospheric moisture through liquid-vapor equilibrium. Although all these mechanisms can be captured using the fully compressible Navier-Stokes equations incorporating energy and water conservation laws coupled with a condensation model, the number of degrees of freedom is expected to be reduced by filtering out specific modes of motion. To tackle this multiscale issue, several mathematical approximations based on the compressible Navier-Stokes equations have been developed e.g. Boussinesq equations [3], pseudo-incompressible equations [4] and the anelastic equations [5]. The Boussinesq approximation is widely adopted in numerical weather prediction tools in which the continuity equation is replaced by an incompressibility condition.

Numerical modelling of atmospheric flows under above mathematical approximations is still challenging due to the large-scale motions and circulations and coupling among multi-physical variables. Accurately modelling the interplay between fluid dynamics and the thermodynamics related to reversible and irreversible moist con-

\footnotetext{
* yongliang.feng@univ-amu.fr

$\dagger$ pierre.sagaut@univ-amu.fr
}

densation or evaporation processes is then key in atmospheric flow modelling. There are two popular numerical treatments of moist microphysics in the Navier-Stokes equations framework [6]. In the first approach, often referred to as the conservative variables (liquid potential temperature and total water mass fraction) approach, the equations of motion are defined using appropriate conservative variables such that terms resulting from phase change are eliminated in the governing equations [7]. This condensation model is mainly employed for shallow convection under Boussinesq approximation. In the second approach, which is more commonly adopted, the equations are again defined using the sensible potential temperature, and separate transport equations for mass fractions of liquid water and vapor water. In the second step a saturation adjustment procedure is performed that correctly allocates the water into its two phases based on the Clausius-Clapeyron formula [8]. Balancing accuracy, efficiency and the physical realisability is problem specific and rarely straightforward. Consequently, there is a continuing quest for improved hydrodynamic core incorporated with condensation models applied in climate, weather and chemistry-transport models [9].

The lattice Boltzmann method (LBM) is an alternative approach to simulate fluid flows based on the Boltzmann equation. Due to its advantages for massively parallel computing as well as its suitability to handle very complex geometries, the LBM has been quickly extended to large scale and multi-scales applications. These applications cover, among others, aerodynamic studies on fullscale vehicles, turbulent flows in urban areas [10], aerodynamic predictions on airfoils [11]. Furthermore, the LBM also has been extended to complex multi-physics phenomena through the additional distribution function 
approach proposes additional conservation law to solve multi-physics fields $[12,13]$. Extending the application scope of lattice Boltzmann method to large-scale meteorological flows by incorporating cloud dynamics has received some interest $[14,15]$, but it is worth noting that most existing LBM approaches for multiphase flows have been assessed considering laminar flows with resolved liquid/gas interfaces (see [16] for a survey) and are therefore not well suited for atmospheric flow simulations.

When the lattice Boltzmann method is applied in complex large-scale geophysical flows [17], such as moist atmospheric convection and condensation, the special limitation on the range of pressure, physical sound speed, relaxation procedure for phase change and associated time and space resolution should be carefully considered, e.g. the numerical instability induced by the use of large time steps, coarse grid and thermal stratification. Besides, the accuracy and efficiency of the condensation models are also highly related with temporal and spatial resolution as well as the numerical schemes [18]. For instance, the numerical stability of the lattice Boltzmann collision model has long been identified among the key issues [19]. In order to overcome the insufficient stability observed in the Bhatnagar-Gross-Krook (BGK) model [20], several improved collision models with enhanced stability have been proposed, e.g. multi-relaxation-time models [21, 22], entropic LB models [23, 24], regularized BGK models [25, 26], cumulant/cascaded LB models [27, 28]. The MRT models were successful in moderately stabilizing the LB method but still remain challenged by high Reynolds numbers $[29,30]$. In addition, their high number of free parameters have motivated the development of alternative models. For example, entropic LB models were developed for turbulent flows through reestablishing $\mathrm{H}$ theorem, which was lost during the discretization [31]. The cumulant and cascaded LB models were developed to avoid the deficits of the MRT models, e.g. violation of Galilean invariance or an insufficient level of hyperviscosity [27, 28]. Moreover, the recursive regularized LB models were designed by considering that the relaxation parameters of the ghosts are chosen to annihilate the higher-order non-equilibrium moments in the post-collision state. The RLB models were successful in simulation of high Reynolds flows [32], even exhibited superconvergence properties for the acoustic problems compared with the MRT models in inviscid limit [33]. In particular, regularized BGK model with hybrid recursive procedure presented both fairly good robustness and accuracy in large scale simulations on urban flows [10, 34].

In this paper, we focus on developing a hybrid recursive regularized lattice Botlzmann method with condensation scheme for cloud dynamics with application to turbulent meteorological flows. The paper is organized as follows. Section II presents governing equations along with the Boussinesq approximation. Section III reviews the lattice Boltzmann method and the approach used to implement the condensation models and to solve humidity and temperature equations, respectively, and the numerical procedure of the proposed method is also presented. Section IV investigates and discusses simulations on a two dimensional double diffusive Rayleigh Bénard convection, as well as a 2D and 3D thermal moist rising bubble under convective atmospheric environment. In particular, the proposed LB approach for humid air and cloud dynamics is assessed considering a shallow cumulus convection in framework of large-eddy simulation. Finally, Section V draws conclusions and perspectives.

\section{MACROSCOPIC PHYSICAL MODEL}

\section{A. Governing equations under the Boussinesq approximation}

The atmosphere is assumed to be a mixture of dry air, water vapor, and liquid water, with respective mass fractions $q_{d}, q_{v}$ and $q_{l}$ ( $q_{v}$ being often referred to as specific humidity). The Boussinesq approximation, widely used for the simulation of atmospheric flows, consists in neglecting density differences everywhere but the gravity terms. Under this assumption, the mass and momentum conservation equations read [6]

$$
\begin{gathered}
\frac{\partial u_{\alpha}}{\partial x_{\alpha}}=0 \\
\frac{\partial u_{\alpha}}{\partial t}+u_{\beta} \frac{\partial u_{\alpha}}{\partial x_{\beta}}=-\frac{1}{\rho_{0}} \frac{\partial p^{\prime}}{\partial x_{\alpha}}+\frac{\partial}{\partial x_{\beta}}\left[\nu\left(\frac{\partial u_{\alpha}}{\partial x_{\beta}}+\frac{\partial u_{\beta}}{\partial x_{\alpha}}\right)\right]+A_{g, z}
\end{gathered}
$$

with use made of Einstein's summation notation. $u_{\alpha}$ represents the components of the velocity vector and $x_{\alpha}$ represents the components of the position vector $(x, y, z)$; $\nu$ is the kinematic viscosity. The hydrodynamic pressure $p^{\prime}=p-p_{0}(z)$ is related to pressure $p$ departing from the height-dependent reference state pressure $p_{0}(z)$ and $\rho_{0}$ the constant reference state density. $A_{g, z}$ is the buoyancy term due to the gravitational acceleration $g$, to be detailed in Eq.(9).

Next, let us define the potential temperature $\theta$ as a function of temperature $T$ following [35]

$$
\Pi=\frac{T}{\theta}=\left(\frac{p_{0}(z)}{p_{0}}\right)^{R_{d} / c_{p}},
$$

where $R_{d}$ is the air gas constant (per mass unit), $c_{p}$ the average mass heat capacity, assumed to be constant and equal to that of dry air.

$$
\Pi=1-\frac{g \cdot z}{c_{p} \cdot \theta_{0}}
$$

is the Exner function [35], $\theta_{0}=T_{0}$ is the reference temperature and $p_{0}$ is the (constant) reference temperature at ground level, not to be confused with $p_{0}(z)$.

With the above definitions, the energy conservation equation may now be expressed in terms of the potential 
temperature $\theta$ as [6].

$$
\frac{\partial \theta}{\partial t}+u_{\alpha} \frac{\partial \theta}{\partial x_{\alpha}}=\frac{\partial}{\partial x_{\alpha}}\left(D_{\theta} \frac{\partial \theta}{\partial x_{\alpha}}\right)+\frac{L_{v} \theta}{c_{p} T} \dot{Q},
$$

where $D_{\theta}$ is the heat diffusion coefficient and $\dot{Q}$ is the mass transfer between the liquid and gas phases associated to $L_{v}$, the mass latent heat of water.

Associated conservation equations for the liquid and vapor mass fractions ( $q_{l}$ and $q_{v}$, respectively, the dry air mass fraction being deduced as $\left.q_{d}=1-q_{v}-q_{l}\right)$ are [6]

$$
\begin{aligned}
\frac{\partial q_{v}}{\partial t}+u_{\alpha} \frac{\partial q_{v}}{\partial x_{\alpha}} & =\frac{\partial}{\partial x_{\alpha}}\left(D_{q} \frac{\partial q_{v}}{\partial x_{\alpha}}\right)-\dot{Q} \\
\frac{\partial q_{l}}{\partial t}+u_{\alpha} \frac{\partial q_{l}}{\partial x_{\alpha}} & =\frac{\partial}{\partial x_{\alpha}}\left(D_{q} \frac{\partial q_{l}}{\partial x_{\alpha}}\right)+\dot{Q}
\end{aligned}
$$

with $D_{q}$ being the water fraction diffusion coefficient.

As to derive the buoyancy acceleration in Eq. (2), let us now define the virtual temperature $\theta_{v}$ as [36]

$$
\theta_{v}=\theta\left[1-\left(1-\frac{1}{\epsilon}\right) q_{v}-q_{l}\right]
$$

with $\epsilon$ the molecular mass ratio of dry air to that of water:

$$
\epsilon=W_{v} / W_{d}=R_{d} / R_{v}
$$

The buoyancy acceleration required in the momentum conservation equation (2) then reads:

$$
A_{g}=\frac{g}{\theta_{\mathrm{v}, H}}\left[\theta_{v}-\theta_{v, H}\right]
$$

where $\theta_{v, H}$ is virtual temperature of reference base state.

\section{B. Phase transition modelling}

In the present work, we assume that the rate of phase transition is infinitely fast, or equivalently, that the liquid and gas phases are in thermo-chemical equilibrium everywhere at all times. Under this assumption, saturation properties provide additional relations between $q_{v}$ and $q_{l}$. The saturation specific humidity is

$$
q_{v}^{s a t}=\frac{\epsilon p_{\mathrm{sat}}}{p_{0}(z)-(1-\epsilon) p_{\mathrm{sat}}}
$$

in which the saturation pressure is approximated following $[37]$

$$
p_{\text {sat }}(T)=610.78 \exp \left[17.269 \frac{T-273.16}{T-35.86}\right],
$$

where $T$ is in Kelvin and $p_{\text {sat }}$ in Pa.

Under the infinitely fast relaxation approximation, the source term $\dot{Q}$ in Eq. (6) can then be computed from

$$
\dot{Q}= \begin{cases}-q_{l} / \delta_{t} & \text { if } q_{v}<q_{v}^{s a t} \text { and } q_{l}<\tilde{\Delta q_{v}} \\ \tilde{\Delta}_{v} / \delta_{t} & \text { otherwise }\end{cases}
$$

where

$$
\tilde{\Delta q_{v}}=\frac{c_{p} R_{d}\left(\theta_{0} \Pi\right)^{2}\left(q_{v}-q_{v}^{s a t}\right)}{c_{p} R_{d}\left(\theta_{0} \Pi\right)^{2}+\epsilon q_{v}^{s a t} L_{v}^{2}\left(\frac{\theta_{0}}{\theta}-\frac{\theta_{0}}{\theta} \frac{R_{d} \theta_{0} \Pi}{\epsilon L_{v}}\right)},
$$

with $\delta_{t}$ being time step following $[6,38]$.

Note that in this framework, Eq. (13) can readily be adapted to address finite rate phase transition, including for instance the liquid droplets size. In the case where only infinitely fast relaxation towards thermochemical equilibrium is of interest, the saturation relation Eq. (10) can be directly included into the conservation equations, thereby reducing the number of partial differential equations, and resulting in a condensation model based on two invariant variables (mass fraction of total water $q_{t}$ and liquid water potential temperature $\theta_{l}$ ). Eq. (6a) and Eq. (6b) can be combined to cancel the source term $\dot{Q}$ in a

$$
q_{t}=q_{v}+q_{l}
$$

conservation equation, becoming

$$
\frac{\partial q_{t}}{\partial t}+u_{\alpha} \frac{\partial q_{t}}{\partial x_{\alpha}}=\frac{\partial}{\partial x_{\alpha}}\left(D_{q} \frac{\partial q_{t}}{\partial x_{\alpha}}\right) .
$$

It then becomes more convenient to rewrite the energy conservation equation using an approximate expression of the liquid water potential temperature [36]

$$
\theta_{l} \approx \theta-\frac{L_{v}}{c_{p} \Pi} q_{l}
$$

to read

$$
\frac{\partial \theta_{l}}{\partial t}+u_{\alpha} \frac{\partial \theta_{l}}{\partial x_{\alpha}}=\frac{\partial}{\partial x_{\alpha}}\left(D_{\theta} \frac{\partial \theta_{l}}{\partial x_{\alpha}}\right),
$$

as to cancel the source term. Where required, $q_{l}$ can then be computed from $q_{t}$ as

$$
q_{l}=\max \left(0, q_{t}-q_{v}^{s a t}\right) .
$$

In that framework, however, $q_{v}^{\text {sat }}$ cannot be evaluated directly from Eq. (10), as $T$ is not available. It is instead approximated from [6]

$$
q_{v}^{s a t}=q^{*} \frac{1+\eta q_{t}}{1+\eta q^{*}}, \quad \eta=\epsilon \frac{L_{\mathrm{v}}^{2}}{c_{p} R_{d} T_{l}^{2}},
$$

where $q^{*}$ corresponds to Eq. (10), evaluated at liquid temperature $T_{l}=\Pi \cdot \theta_{l}$, rather than $\Pi . \theta$. Compared to the former approach, only two equations of invariant variables $\left(\theta_{l}, q_{t}\right)$ need to be solved in the reduced model, and the relaxation step is replaced by a recovery procedure, which is performed using the approximate expression of saturation humidity following Eq. (19). It is worth noting that the set of invariant variables $\left(\theta_{l}, q_{t}\right)$ could often be simply derived in the shallow convection.

In the following, the model based on set of governing equations about invariant variables $\left(q_{t}, \theta_{l}\right)$ is referred to as 1eq. model, whereas the model based on the variables $\left(q_{l}, q_{v}, \theta\right)$ is referred to as the 2eq. model, as summarized in Table $I$. 
TABLE I. Governing equations for the 2eq. and 1eq. models.

\begin{tabular}{l|c|c}
\hline \hline Governing equations & 2eq. model & 1eq. model \\
\hline Mass conservation & Eq. (1) & Eq. (1) \\
Momentum conservation & Eq. (2) & Eq. (2) \\
Energy conservation & Eq. (5) & Eq. (17) \\
Water conservation & Eq. (6a) and Eq. (6b) & Eq.(15) \\
\hline \hline
\end{tabular}

\section{HYBRID RECURSIVE REGULARIZED LATTICE BOLTZMANN MODEL}

This section presents the hybrid recursive regularized Lattice Boltzmann model (HRR-LBM) proposed to solve the governing equations. The idea is to solve the mass and momentum equations $(1,2)$ following a LB approach [39], whereas the energy and water conservation(s) equation(s) are solved by a classical finite volume (FV) approach. The present developments have been performed in the ProLB solver.

\section{A. Lattice Boltzmann solver}

\section{Reminders about $L B M$}

Lattice Boltzmann methods aim at solving the lattice Boltzmann equation through space, time, and velocity discretization [39, 40]. Space and time are classically discretized on a cartesian grid, whereas speeds are discretized on a so-called $\mathrm{D} n \mathrm{Q} m$ lattice ( $n$ dimensions and $m$ discrete velocities $\boldsymbol{c}_{i}$ ).

The flow problem is then solved for $f_{i}(\boldsymbol{x}, t)$, the density distribution of particles with velocity $\boldsymbol{c}_{i}$ at $(\boldsymbol{x}, t)$, which can be obtained at time $t+\delta_{t}$ through the so-called BGK collision model [40]

$$
\begin{aligned}
f_{i}\left(\boldsymbol{x}+\boldsymbol{c}_{i} \delta_{t}, t+\delta_{t}\right) & =f_{i}(\boldsymbol{x}, t)-\frac{1}{\tau}\left[f_{i}(\boldsymbol{x}, t)-f_{i}^{e q}(\boldsymbol{x}, t)\right] \\
& +\left(1-\frac{1}{2 \tau}\right) \delta_{t} F_{i}(\boldsymbol{x}, t),
\end{aligned}
$$

a succession of a streaming and a collision step [41, 42] which is equivalent to a Strang-splitting-based time integration method [43]. The characteristic collision time $\tau$ is related to the kinematic viscosity $\nu$ through $\nu=$ $c_{s}^{2}\left(\tau-\frac{1}{2}\right) \delta_{t}$, and $c_{s}$ is the lattice sound speed, inherent of the lattice $\mathrm{D} n \mathrm{Q} m$ choice [42]. Density and momentum can then be recovered as

$$
\begin{aligned}
\rho(\boldsymbol{x}, t) & =\sum_{i=1}^{m} f_{i}(\boldsymbol{x}, t), \\
\rho \boldsymbol{u}(\boldsymbol{x}, t) & =\sum_{i=1}^{m} \boldsymbol{c}_{i} f_{i}(\boldsymbol{x}, t)+\frac{\delta_{t}}{2} \sum_{i=1}^{m} \boldsymbol{c}_{i} F_{i}(\boldsymbol{x}, t),
\end{aligned}
$$

where $F_{i}(\boldsymbol{x}, t)$ is an external force [44].

\section{The recursive regularized Lattice-Boltzmann solver}

Considering that the third-order equilibrium distribution function and recursive regularized collision model exhibit considerably better performance on numerical stability and accuracy compared to the original LBGK model [17, 45-49], a recursive regularized collision model associated with the third-order equilibrium distribution function is employed as the collision model. The lattice Boltzmann equation Eq. (20) is rewritten as

$f_{i}\left(\boldsymbol{x}+\boldsymbol{c}_{i} \delta_{t}, t+\delta_{t}\right)=f_{i}^{e q}(\boldsymbol{x}, t)+\left(1-\frac{1}{\tau}\right) f_{i}^{n e q}(\boldsymbol{x}, t)+\frac{\delta_{t}}{2} F_{i}$

where $f_{i}^{n e q}=f_{i}-f_{i}^{e q}+\delta_{t} F_{i} / 2$ is off-equilibrium distribution function associated with the third-order equilibrium distribution function $f_{i}^{e q}[45,47,50]$

$$
\begin{aligned}
& f_{i}^{e q}=w_{i}\left[\rho+\frac{c_{i \alpha} \rho u_{\alpha}}{c_{s}^{2}}+\frac{a_{0, \alpha \beta}^{(2)} \mathcal{H}_{i \alpha \beta}^{(2)}}{2 c_{s}^{4}}+\frac{a_{0, \alpha \beta \gamma}^{(3)} \mathcal{H}_{i \alpha \beta \gamma}^{(3)}}{6 c_{s}^{6}}\right], \\
& a_{0, \alpha \beta}^{(2)}=\rho u_{\alpha} u_{\beta}, \quad \mathcal{H}_{i \alpha \beta}^{(2)}=c_{i \alpha} c_{i \beta}-c_{s}^{2} \delta_{\alpha \beta}, \\
& a_{0, \alpha \beta \gamma}^{(3)}=\rho u_{\alpha} u_{\beta} u_{\gamma}, \quad \mathcal{H}_{i \alpha \beta \gamma}^{(3)}=c_{i \alpha} c_{i \beta} c_{i \gamma}-c_{s}^{2}[c \delta]_{\alpha \beta \gamma} .
\end{aligned}
$$

where $\mathcal{H}_{i, \alpha \beta}$ and $\mathcal{H}_{i, \alpha \beta \gamma}$ correspond to the second and third order Hermite polynomials. The corresponding offequilibrium distribution function $f_{i}^{n e q}$ is also expended in Hermite polynomial according to Chapman-Enskog technique as

$$
f_{i}^{n e q}=\frac{a_{1, \alpha \beta}^{(2)} \mathcal{H}_{i \alpha \beta}^{(2)}}{2 c_{s}^{4}}+\frac{a_{1, \alpha \beta \gamma}^{(3)} \mathcal{H}_{i \alpha \beta \gamma}^{(3)}}{6 c_{s}^{6}},
$$

and reconstructed by a Recursive Regularized BGK Lattice-Boltzmann Method (RR-BGK-LBM) with forcing terms as

$$
\begin{gathered}
a_{1, \alpha \beta}^{(2)}=\sum_{i} \mathcal{H}_{i, \alpha \beta}^{(2)}\left(f_{i}-f_{i}^{e q}+\frac{\delta_{t}}{2} F_{i}\right), \\
a_{1, \alpha \beta \gamma}^{(3)}=u_{\alpha} a_{1, \beta \gamma}^{(2)}+u_{\beta} a_{1, \gamma \alpha}^{(2)}+u_{\gamma} a_{1, \alpha \beta}^{(2)} .
\end{gathered}
$$

Lastly, to account for the buoyancy force Eq. (9) in the momentum equation (2), the external force term $F_{i}(\boldsymbol{x}, t)$ in Eq. (22) is prescribed $[44,51]$ as

$$
F_{i}=\rho w_{i}\left[\frac{A_{\alpha} c_{i \alpha}}{c_{s}^{2}}+\frac{\left(u_{\alpha} A_{\beta}+u_{\beta} A_{\alpha}\right)\left(c_{i \alpha} c_{i \beta}-c_{s}^{2} \delta_{\alpha \beta}\right)}{2 c_{s}^{4}}\right] .
$$

Through the Chapman-Enskog technique [39], it can be shown that the lattice Boltzmann BGK equation (22), appended with the expression for the equilibrium (23) and non-equilibrium (24) functions, and the forcing term (27) recovers the mass (1) and momentum (2) conservation equations in the low-Mach regime.

In the simulations, physical and lattice units for length and time are related through a reference length scale $L_{0}$, a physical reference sound speed $c_{s p}$ for space and time. 
Accordingly, the physical variables can be converted from the quantities in lattice units ( $l$ subscript) as

$$
\begin{gathered}
\delta_{x}=L_{0} / N_{n}, \quad \delta_{t}=\delta_{x} \cdot \frac{c_{s}}{c_{s p}}, \quad t=N_{t} \cdot \delta_{t} \\
x=N_{i} \cdot \delta_{x}, \quad u=u_{l} \cdot \frac{\delta_{x}}{\delta_{t}}, \quad \nu=\nu_{l} \frac{\delta_{x}^{2}}{\delta_{t}}
\end{gathered}
$$

The physical reference sound speed $c_{s p}$ is kept as a free parameter. This allows to freely accelerate the convergence rate, by varying the time step in the same manner of Courant-Friedrichs-Lewy (CFL) number for conventional Navier-Stokes solvers.

\section{B. Finite Volume solver}

Numerically, the multiple distribution function model for scalar transport equation is not optimal from the computational standpoint, even though this numerical inefficiency can be improved somewhat by using some redundant degree of freedom in LB models in diffusion dominated condition [52]. Besides, it is convenient to use an upwind biased scheme to preserve the numerical stability in meteorological flows in which the strong advection effect and stiff terms due to phase transition commonly exist. Energy and water conservation equations (5, 6a, 6b, 15 and 17) of both the 2eq. and 1eq. models (see Tab. I) are solved with a finite volume method. They may all be written under the non-conservative generic form

$$
\frac{\partial \phi}{\partial t}+u_{\alpha} \frac{\partial \phi}{\partial x_{\alpha}}=\frac{\partial}{\partial x_{\alpha}}\left(D \frac{\partial \phi}{\partial x_{\alpha}}\right)+\Psi
$$

where $\phi$ is the scalar (e.g. $q_{l}, \theta_{l}, \ldots$ ), $D$ is the diffusion coefficient, provided via specification of Prandtl numbers

$$
\operatorname{Pr}_{\theta}=\nu / D_{\theta}, \quad \operatorname{Pr}_{q}=\nu / D_{q},
$$

for the heat and molecular diffusion, respectively. $\Psi$ is a source term (e.g. related to the phase transfer rate $\dot{Q})$, and velocity $u_{\alpha}$ required in the convective term is obtained from the LB solver via Eq. (21).

In the present hybrid LB approach, the scalars transport equations e.g. temperature, humidity are solved by a vertex based finite volume (FV) method in the same temporal-spatial discrete coordinates of LB method. The coupling between LB and FV is detailed in Fig. 1. The first-order explicit Euler scheme is adopted as temporal discretization, which is given as

$$
\phi^{n+1}=\phi^{n}+\delta_{t}\left[\operatorname{RHS}_{C}\left(\phi^{n}\right)+\operatorname{RHS}_{D}\left(\phi^{n}\right)+\Psi^{n}\right]
$$

where $\mathrm{RHS}_{C}, \mathrm{RHS}_{D}$ represents convection term and diffusion term, respectively. The convective flux is constructed using MUSCL scheme [53] with the van Albada limiter [54]. The classical second-order central difference scheme is adopted for the diffusion term.

\section{Implementation of boundary conditions}

The boundary conditions of distribution function are implemented by a finite difference reconstruction approach along with a regularization procedure $[25,55]$.

1. First, the macroscopic velocities $\boldsymbol{u}$ on the boundary nodes are prescribed in the case of a Dirichlet boundary condition or extrapolated in the case of a Neumann boundary condition.

2. Next, the shear stress tensor is computed using the velocity gradients on boundary nodes, which are computed on these nodes using a first order biased scheme.

3. Then, the density distribution function is reconstructed by Eq. (23) and off-equilibrium moment as follows

$$
\begin{aligned}
a_{1, \alpha \beta}^{(2)} & \approx-\rho \tau c_{s}^{2}\left(\frac{\partial u_{\alpha}}{\partial x_{\beta}}+\frac{\partial u_{\beta}}{\partial x_{\alpha}}\right), \\
a_{1, \alpha \beta \gamma}^{(3)} & =u_{\alpha} a_{1, \beta \gamma}^{(2)}+u_{\beta} a_{1, \gamma \alpha}^{(2)}+u_{\gamma} a_{1, \alpha \beta}^{(2)},
\end{aligned}
$$

It is worth noting that the density values on the bottom surface node are set to constant reference density and densities on other boundary are extrapolated from inside nodes.

4. For humidity and temperature fields which are solved by the finite volume approach, the macroscopic values $\theta$ or $\theta_{l}$ and $q_{t}$ or $\left(q_{v}, q_{l}\right)$ are simply prescribed or extrapolated on all boundary nodes.

\section{Summary of the algorithm}

The algorithm consists of a LB and a FV solver running simultaneously. The only data exchanges between the two solvers are :

- the velocity $\boldsymbol{u}$ obtained from Eq. (21) in the LB solver and sent to the FV solver to assess convective fluxes,

- $\theta_{v}$, obtained from the energy equation in the FV solver, and sent to the LB solver to update the forcing term.

The complete algorithm is detailed in Fig. 1 in the case of the 2eq. model, the 1eq model merely being a reduction of the former. The 2D (resp. 3D) computational examples presented in the next section were obtained within the D2Q9 (resp. D3Q19) framework in ProLB $[56]$. 


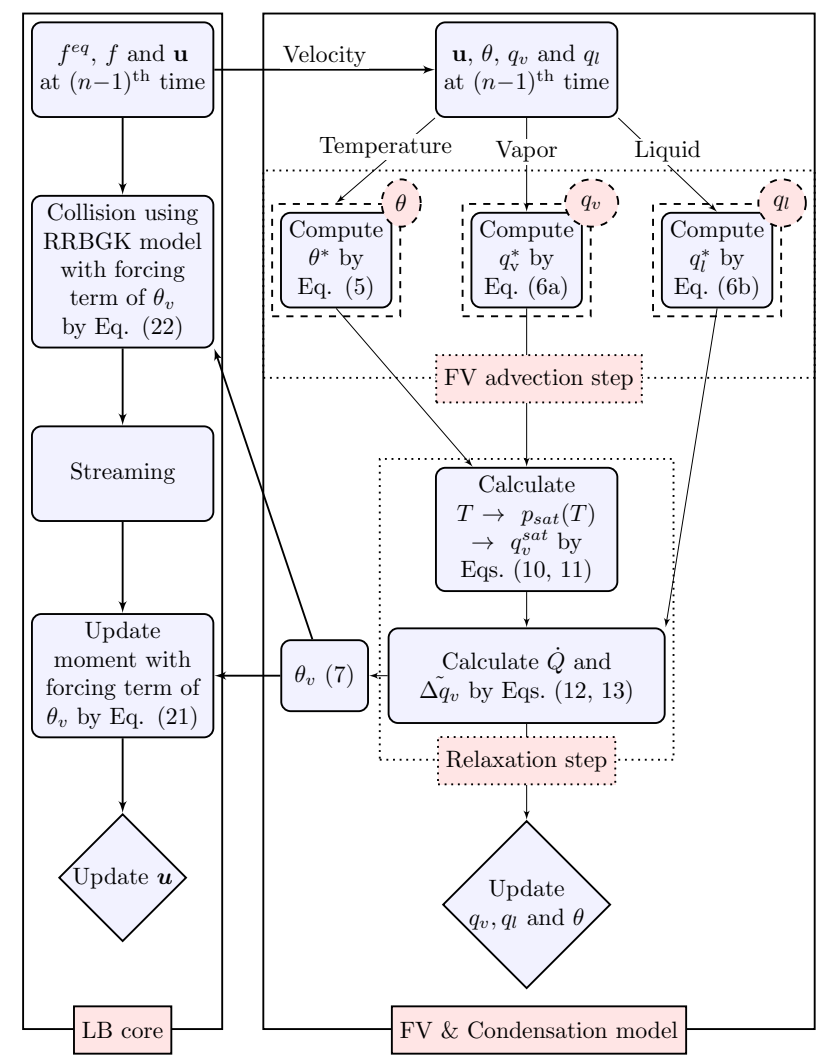

FIG. 1. The flowchart of LB method with condensation model (2eq). (potential temperature $\theta$, vapor humidity $q_{v}$ and cloud liquid specific humidity $q_{l}$ ).

\section{RESULTS AND DISCUSSION}

\section{A. Double diffusive Rayleigh Bénard convection}

Albeit not technically a moist convection condensation problem, a two dimensional Rayleigh-Bénard convection is studied in a square domain with height of $H=1 \mathrm{~m}$ to assess the proposed LB method on coupling of temperature, humidity and velocity. The Rayleigh-Bénard convection is regarded as a classical motion of atmospheric flows under convective stratification. In moist atmosphere, the humid air is heated in the hot ground surface and cooled in the cold top boundary. Density variations of humid air caused by the temperature and humidity variations drive the convection flow under gravity acceleration while the viscosity will counteract to equilibrate the motion which is simplified and modeled as a double diffusive Rayleigh-Bénard convection.

For this test case, we use the 2eq. model where the condensation term is turned off and heat specific capacity $c_{p}$ is set to infinity. Thus the Exner function $\Pi$ is set to unity and the following temperatures are equal: $\theta=\theta_{l}=$ $T$. In addition, the virtual temperature (7) is modified
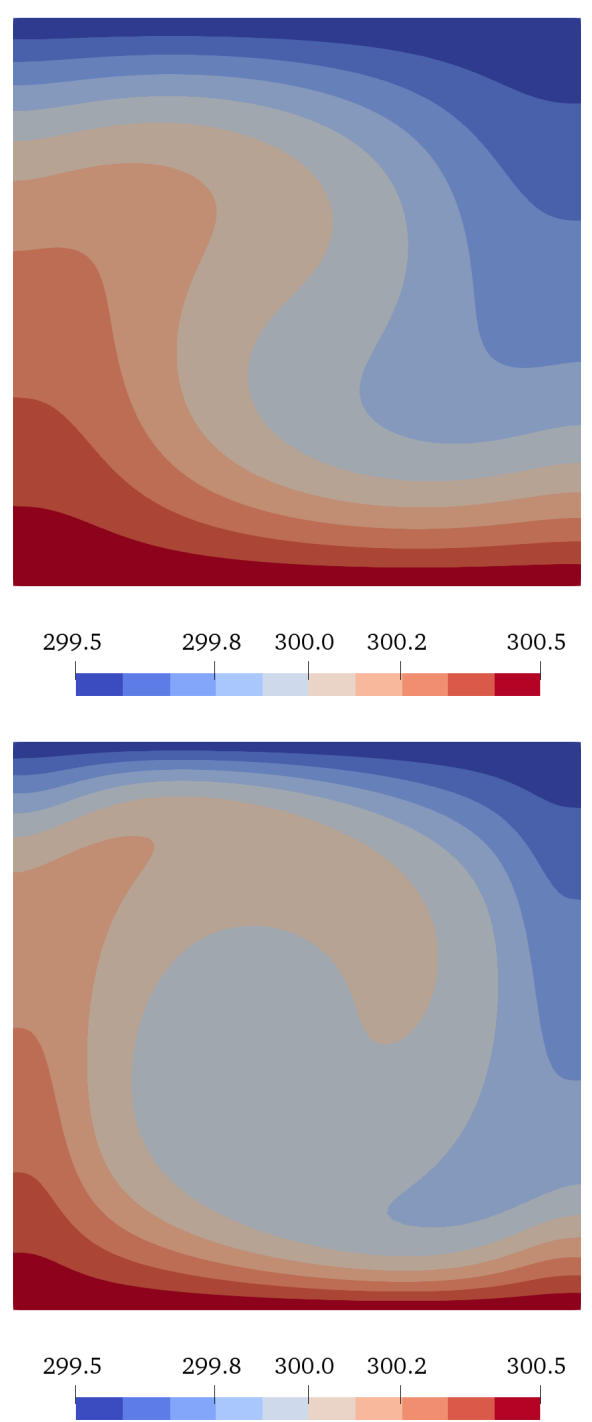

FIG. 2. Temperature contours obtained by the LB method (2eq. model) for $R a=10^{4}$ (top), and $R a=10^{5}$ (bottom).

as

$$
\theta_{v}=\theta\left[1-\left(1-\frac{1}{\epsilon}\right)\left(q_{l}+q_{v}\right)\right]
$$

in the buoyancy force (9) as to account only for the total humidity $q_{t}=q_{v}+q_{l}$. The Prandtl number $\operatorname{Pr}_{\theta}$ and $\operatorname{Pr}_{q}$ are both set to 0.71 for air. The enclosure boundary conditions are non-slip. The bottom and top surface is respectively at a constant temperature $299.5 \mathrm{~K}$ and 300.5 $\mathrm{K}$. The specific humidity is set to $q_{t}=0.003\left(q_{l}=0.001\right.$, $\left.q_{v}=0.002\right)$ and $q_{t}=0\left(q_{l}=0, q_{v}=0\right)$ at the bottom and top boundary, respectively. Adiabatic conditions for both temperature and humidity are set on the right and left boundaries.

The Rayleigh number is defined as

$$
\mathrm{Ra}=\operatorname{Pr}_{\theta} \frac{g H^{3} \Delta \theta_{v}}{\nu^{2} \theta_{0}}
$$



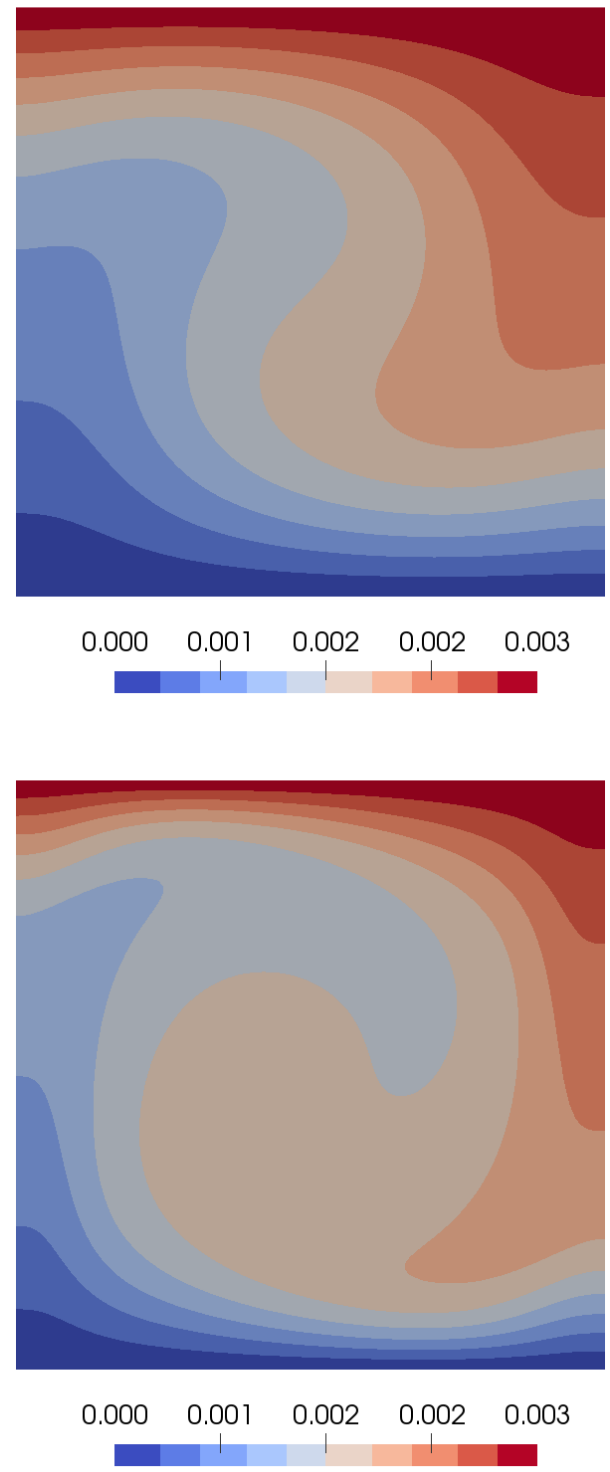

FIG. 3. Contours of water fraction obtained by the LB method (2eq. model) on $R a=10^{4}$ (top), $R a=10^{5}$ (bottom).

where $\theta_{0}$ is $300.5 \mathrm{~K}$ and kinematic viscosity $\nu$ is calculated by Ra given in the simulations. Simulation of double diffusive Rayleigh Bénard convection with $\mathrm{Ra}=10^{4}$ and $10^{5}$ are presented hereafter, on $50 \times 50$ and $100 \times 100$ grids. An equivalent Reynolds number can be computed as $\operatorname{Re}=\sqrt{\mathrm{Ra} / \mathrm{Pr}_{\theta}}$, which approximately equals 119 and 375 , respectively. The time step is calculated by $\delta_{t}=\left(c_{s} / c_{s p}\right) \delta_{x}$ with a sound speed $c_{s p}=1 \mathrm{~m} \mathrm{~s}^{-1}$. For $\mathrm{Ra}=10^{4}$, the non-dimensional relaxation time $\tau$ is about to 0.589 and 0.678 , respectively. For $\mathrm{Ra}=10^{5}$, the value of $\tau$ is approximately equal to 0.528 and 0.556 .

Figure 2 and Fig. 3 respectively display the contours of potential temperature and total water humidity when the steady state has been achieved. It is seen that both the temperature field and water field obtained by the hybrid LB model are in good agreement with results displayed in [57]. The reference solution [57] was obtained using a finite-volume method discretized using QUICK scheme in the momentum equation and a second order central differencing one in the energy equation on a non-uniform grid with $256^{2}$ grids.
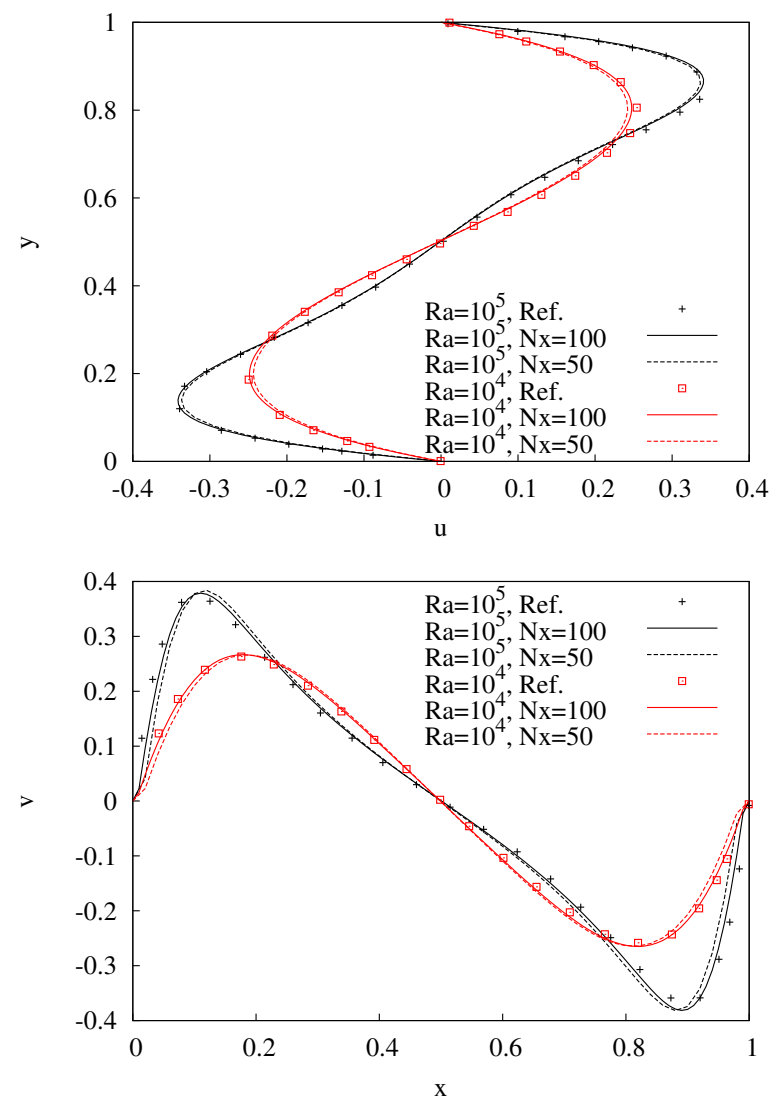

FIG. 4. Profile of velocity along midline of domain (top: velocity $u$ along $y$; bottom: velocity $v$ along $x$ ). The double diffusive Rayleigh Bénard convection with $R a=10^{4}$ and $10^{5}$ are presented on $50 \times 50$ and $100 \times 100$ grid resolutions by the proposed LB method.

A comparison between the results obtained by the present LB model and the results in [57] is reported in Fig. 4. Both the horizontal velocity $u$ through vertical middle line of square enclosure and the vertical velocity $v$ through horizontal center line are plotted and compared in Fig. 4. Both components of the velocity are in very good agreement with the data in literature, indicating that the hybrid recursive regularized LB model can be accurately applied in double diffusive convection problems.

For further validation, the different $\tau$ associated with various values of gravity g, e.g., $9.8,0.98,0.098 \mathrm{~m} \mathrm{~s}^{-2}$ in Eq. (35) are investigated in the convection at $\mathrm{Ra}=10^{4}$ on $50 \times 50$ grids. The comparison on horizontal and vertical velocities is reported in Fig 5 . It can be found that the excellent agreement is obtained by the present method 


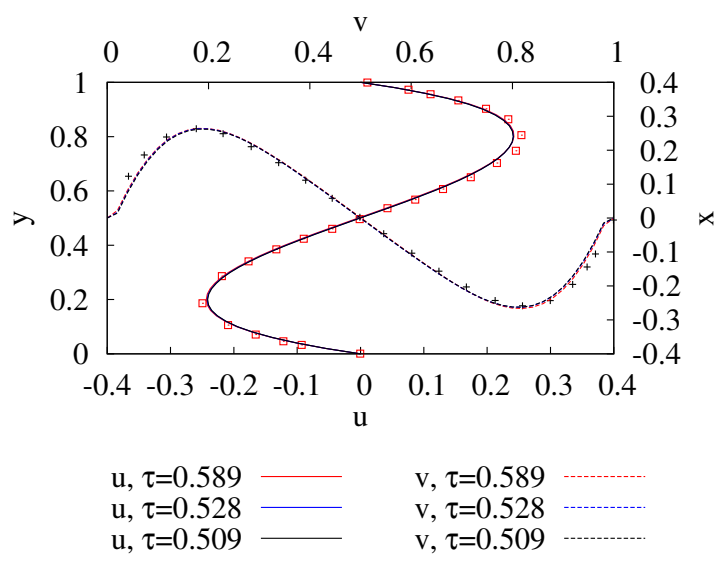

FIG. 5. Comparison of velocities (u, v) along midlines of domain with different non-dimensional relaxation time $\tau$ at $\mathrm{Ra}=10^{4}$ on $50 \times 50$ grids.

with different values of $\tau$.

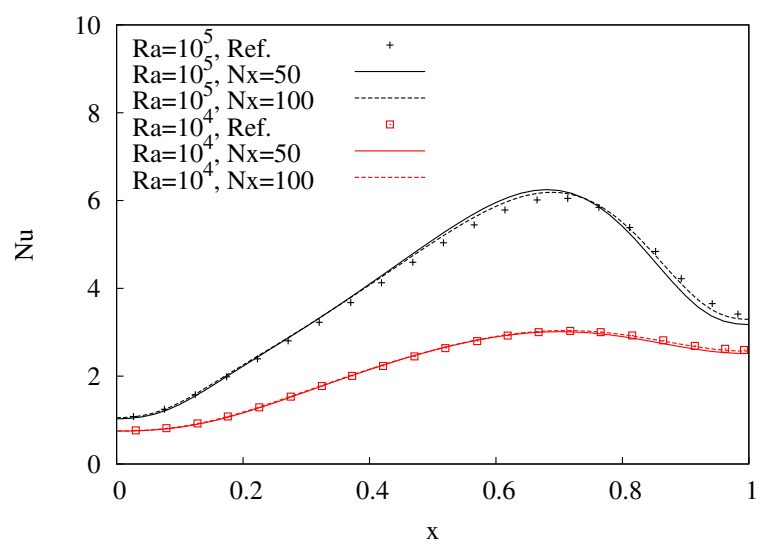

FIG. 6. Local Nusselt number through the hot wall. The double diffusive Rayleigh Bénard convection with $\mathrm{Ra}=10^{4}$ and $10^{5}$ are presented on $50 \times 50$ and $100 \times 100$ grid resolutions by the proposed LB method. ber

Figure 6 reports a comparison of the local Nusselt num-

$$
N u(x)=\left.\frac{L}{\left(\theta_{v, H}-\theta_{v, 0}\right)} \frac{\partial \theta_{v}}{\partial x}\right|_{\text {bottom }},
$$

as obtained with the present and in [57], showing an excellent quantitative agreement.

\section{B. A two-dimensional rising moist thermal bubble}

A rising moist thermal bubble in atmosphere is examined using the present LB model. The simulation is twodimensional, with a domain height of $2.4 \mathrm{~km}$ and width of $3.6 \mathrm{~km}$. Periodic boundary conditions are set on either side of the domain and symmetric nonconducting boundary at the top and bottom surfaces where

$$
\frac{\partial u}{\partial z}=0, \frac{\partial^{2}}{\partial z^{2}}\left(q_{v}, q_{l}, \theta\right)=0, \text { at } z=0, H
$$

The initial unperturbed environment is calm (zero initial wind everywhere) and defined by a constant stratification effect, which is introduced by the Brunt-Väisälä frequency,

$$
N=\sqrt{\frac{g}{\theta} \frac{\mathrm{D} \theta}{\mathrm{D} z}} \approx 0.0113 \mathrm{~s}^{-1} .
$$

A constant relative humidity of 20 percent is given as a base state humid environment with a surface temperature $\theta_{0}$ and pressure $p_{0}$ of $283 \mathrm{~K}$ and $85,000 \mathrm{~Pa}$. Therefore, a circular perturbation only on relative humidity is centered in the domain at $z=0.8 \mathrm{~km}$. The relative humidity (RH) is set to 100 percent from radius $r \leq 200 \mathrm{~m}$. For $200 \mathrm{~m} \leq r \leq 300 \mathrm{~m}$, a transition layer is assumed in which

$$
\mathrm{RH}=20 \%+80 \% \cos ^{2}\left(\frac{\pi}{2} \frac{r-200}{100}\right)
$$

The vapor specific humidity $q_{v}$ is converted from

$$
q_{v}=\mathrm{RH} \frac{\epsilon \cdot p_{\text {sat }}}{p_{0}-(1-\epsilon) p_{\text {sat }}}
$$

The kinematic viscosity $\nu$ is assigned $1 \mathrm{~m}^{2} \mathrm{~s}^{-1}$ and Prandtl number $\operatorname{Pr}_{\theta}$ and $\operatorname{Pr}_{q}$ are both set to unity in the simulations. The simulations based on the present hybrid recursive regularized lattice Boltzmann method with condensation model (1eq) and (2eq) are carried with $\delta \mathrm{x}=5 \mathrm{~m}$. The time step is calculated by $\delta_{t}=\left(c_{s} / c_{s p}\right) \delta_{x}$ with a sound speed in physical unit $c_{s p}=85 \mathrm{~m} \mathrm{~s}^{-1}$. Thus, the non-dimensional relaxation time $\tau \approx 0.504$ is used in the simulation. Results of the simulations are presented in Fig. 7. Due to the symmetry of the case tested, the streamlines and mass fraction of liquid water obtained by 2eq model are plotted in the left sub-domain (1.1-1.8 km) while the solution calculated from 1eq model is presented in right sub-domain. In excellent agreement with the results in Ref.[see 58, Fig.6], the warm moist bubble rises and expands over time. The phase transition from vapor to liquid gradually happens, while the bubble rises. The benchmark solution in [58] was obtained with a multidimensional positive definite advection transport algorithm on $2.5 \mathrm{~m}$ grid spacing.

In order to quantitatively validate the proposed LB model, the highest vertical position of contour of $20 \%$ maximum $q_{l}$ (denoted as $H_{20}$ ) and vertical fluid velocity on the top central position of interface (denoted as $\left.W_{f}\right)$ are given in Table II. The computed velocities at 3, 5 and 7 min are in good agreement with benchmark solution given in Ref. [58]. The benchmark solution was obtained using an anelastic flow solver using variables $\left(\theta, q_{v}, q_{l}\right)$ on $2.5 \mathrm{~m}$ grid spacing. The vertical position of contour of $20 \%$ maximum $q_{l}$ reported by our LB models is consistent with reference values at $3 \mathrm{~min}$. However, 

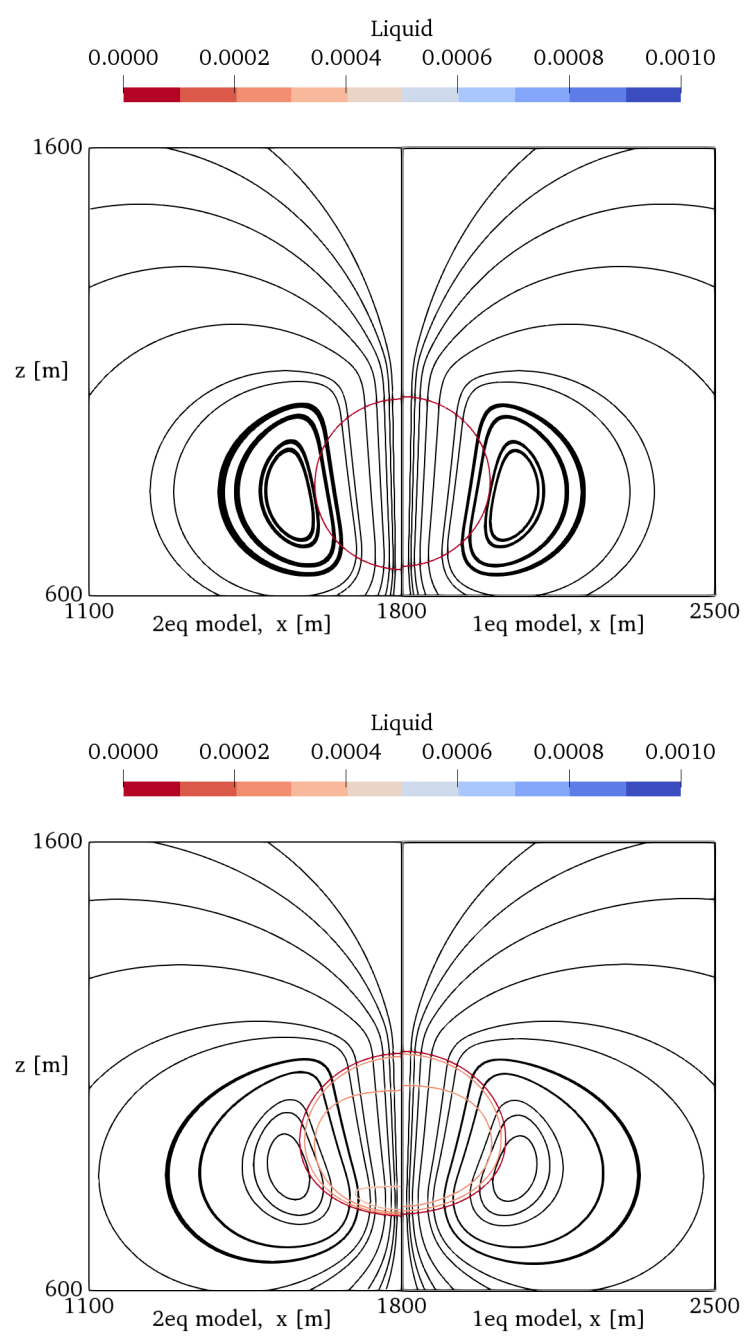

FIG. 7. Part I: Streamlines and contours of liquid humidity field at $2 \mathrm{~min}$ (top) and $3 \mathrm{~min}$ (bottom) obtained by the present LB method with 1eq model and 2eq model. In each figure, the left part is obtained by 2 eq model while the right part is from leq model.

the deviation on the vertical location between our models and reference values gradually increases with physical time. This deviation may be the consequence of Boussinesq approximation which is used in our model rather than the anelastic approximation adopted in Ref. [58].

To further investigate numerical stability of the LB model, random perturbations with a small amplitude are introduce during the simulation as same as calculations in Ref. [58]. An excitation at $t=3$ min was provided by adding a perturbation to potential temperature $\theta$ for 1 eq model $\theta_{l}$ for 2 eq model which ranged between $\pm 0.05 \mathrm{~K}$ on every node.

Figure 8 and Fig. 9 show contours of vorticity and liquid humidity field after excitation of instability on resolution of $5 \mathrm{~m}$ at $7 \mathrm{~min}$ and $10 \mathrm{~min}$, respectively. The red solid lines represents vorticity from $-0.16 \mathrm{~s}^{-1}$ to $-0.02 \mathrm{~s}^{-1}$
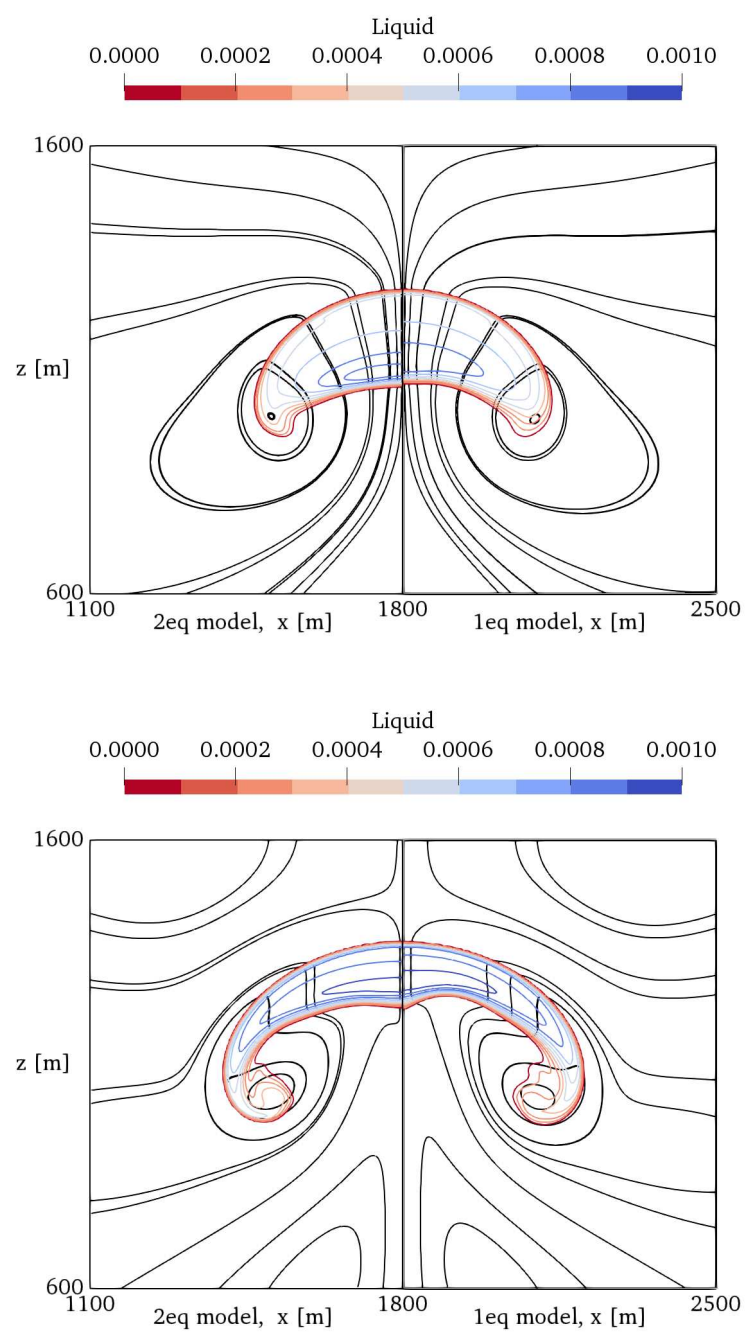

FIG. 7. Part II: Streamlines and contours of liquid humidity field at $5 \mathrm{~min}$ (top) and $7 \mathrm{~min}$ (bottom) obtained by the present LB method with 1eq model and 2eq model. In each figure, the left part is obtained by 2 eq model while the right part is from 1eq model.

with interval of $0.07 \mathrm{~s}^{-1}$ while the blue solid lines represents vorticity from $0.02 \mathrm{~s}^{-1}$ to $0.16 \mathrm{~s}^{-1}$ with the same interval. The cloud map shows mass fraction of liquid water in domain of $[1100 \mathrm{~m}, 2500 \mathrm{~m}] \times[800 \mathrm{~m}, 1800 \mathrm{~m}]$ . The similar instability on cloud formatting is observed with comparison of the results in Ref.[see 58, Fig.15]. Besides, a slight different of cloud formatting is obtained by 1 eq model and 2 eq model at $10 \mathrm{~min}$. The 2 eq model obtained a little more complex structure of vorticity and cloud when the bubble rose to the high altitude, which is consistent with the solution in [58]. A complex instability was excited in their results which was obtained by two equation condensation model using monotone finite difference method on $2.5 \mathrm{~m}$ resolution. The difference between our two results and the difference between our results and their solution could be caused by different 
TABLE II. The highest vertical location of contour of $20 \%$ maximum $q_{l}\left(H_{20}\right)$ and vertical fluid velocity on the top central position of interface $\left(W_{f}\right)$ at 3,5 and $7 \mathrm{~min}$.

\begin{tabular}{lllll}
\hline \hline physical time & variables & 2eq. & 1eq. & Ref. \\
\hline \multirow{2}{*}{3 min } & $H_{20}[\mathrm{~m}]$ & 1128 & 1133 & 1194 \\
& $W_{f}\left[\mathrm{~m} \mathrm{~s}^{-1}\right]$ & 1.60 & 1.59 & 1.59 \\
\hline \multirow{2}{*}{$5 \mathrm{~min}$} & $H_{20}[\mathrm{~m}]$ & 1278 & 1281 & 1363 \\
& $W_{f}\left[\mathrm{~m} \mathrm{~s}^{-1}\right]$ & 1.21 & 1.24 & 1.21 \\
\hline \multirow{2}{*}{$7 \mathrm{~min}$} & $H_{20}[\mathrm{~m}]$ & 1374 & 1373 & 1468 \\
& $W_{f}\left[\mathrm{~m} \mathrm{~s}^{-1}\right]$ & 0.56 & 0.52 & 0.72 \\
\hline \hline
\end{tabular}
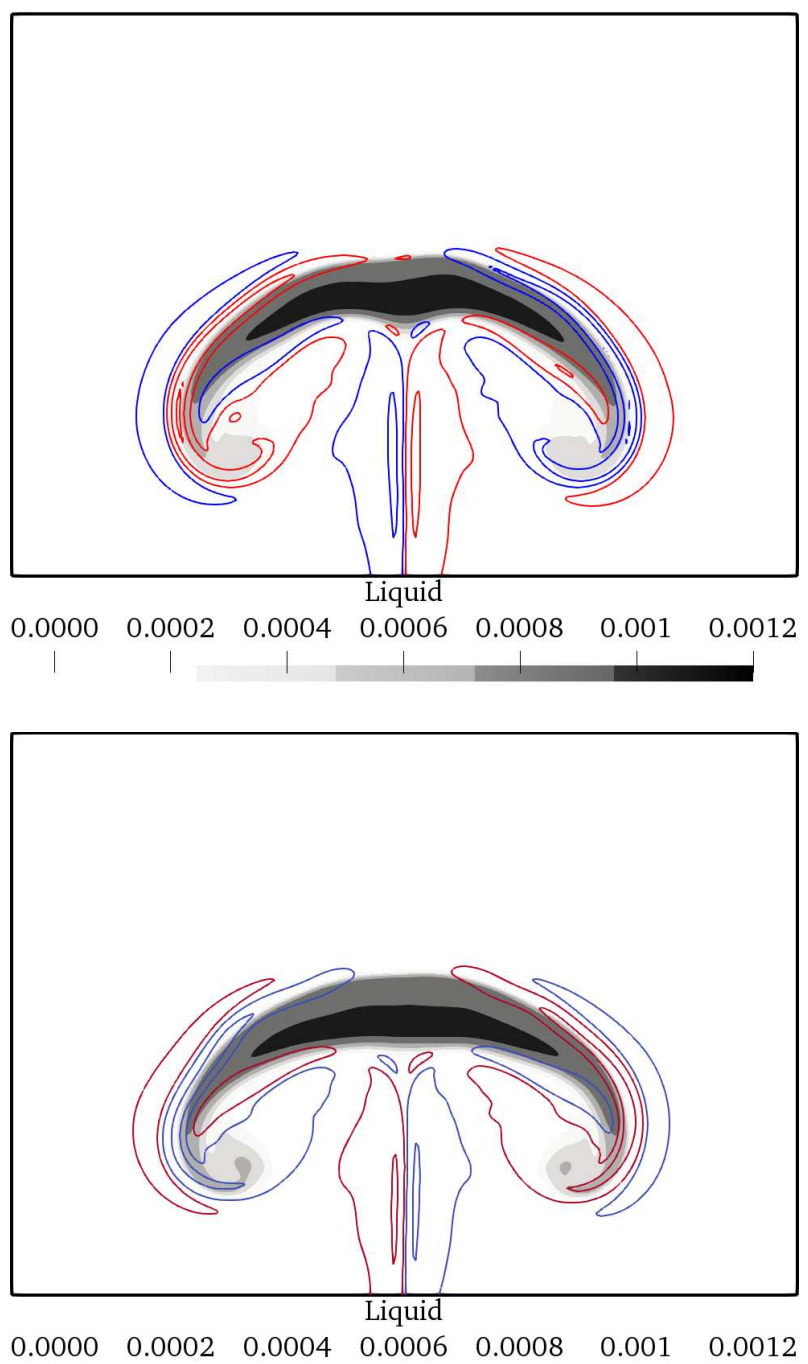

FIG. 8. Contours of vorticity and liquid humidity field obtained by 1eq model (top) and 2eq model (bottom) at 7 min with excitation of instability on resolution of $5 \mathrm{~m}$ in domain of $[1100 \mathrm{~m}, 2500 \mathrm{~m}] \times[800 \mathrm{~m}, 1800 \mathrm{~m}]$.
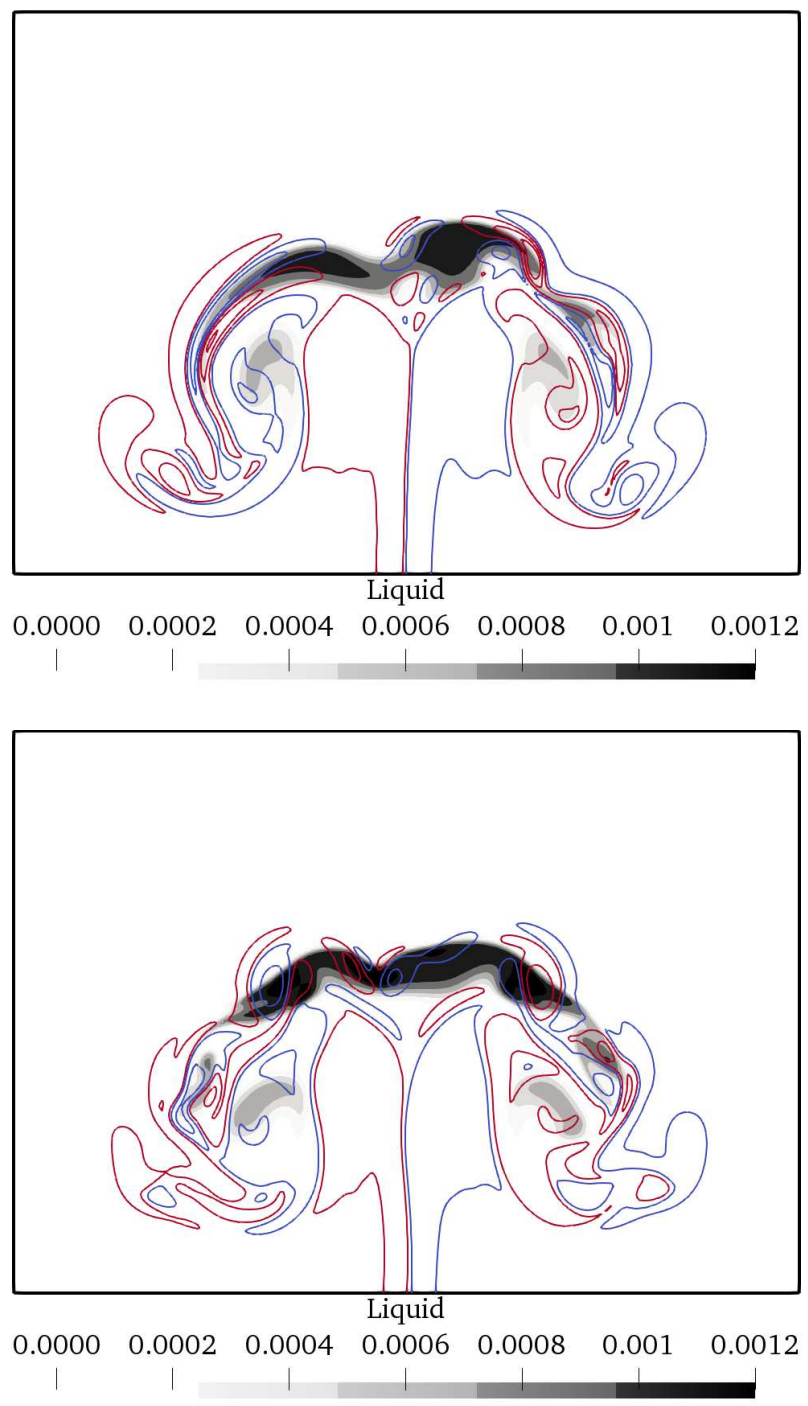

FIG. 9. Contours of vorticity and liquid humidity field obtained by 1eq model (top) and 2eq model (bottom) at $10 \mathrm{~min}$ with excitation of instability on resolution of $5 \mathrm{~m}$ in domain of $[1100 \mathrm{~m}, 2500 \mathrm{~m}] \times[800 \mathrm{~m}, 1800 \mathrm{~m}]$.

numerical characteristics of two methods, which strongly influenced the interactions between numerical noises and excitation instability. Considering the 2eq model is more general in both shallow and deep atmospheric flows and that it can be conveniently extended for involving the precipitation microphysics, only the 2 eq model is used in the following study.

\section{A three-dimensional rising moist thermal bubble}

The simulation of the rising moist thermal bubble is extend to three-dimensional study in this section. The investigated domain is three-dimensional, with a domain 
height of $2.4 \mathrm{~km}$, length of $3.6 \mathrm{~km}$ and width of $3.6 \mathrm{~km}$. Periodic boundary conditions are specified four laterals of the domain and free slip nonconducting boundary at the top and bottom surfaces, which is the same with the setting in previous 2D simulation. The same initial unperturbed environment is adopted and defined by the constant stratification effect. A sphere perturbation only on relative humidity is centered in the domain at $z=0.8 \mathrm{~km}$. The relative humidity $(\mathrm{RH})$ is set to 100 percent from radius $r \leq 200 \mathrm{~m}$. For $200 \mathrm{~m} \leq r \leq 300 \mathrm{~m}$, a similar transition layer is assumed with the one in the previous $2 \mathrm{D}$ case.

TABLE III. Setup of grid refinement for simulation of 3D rising moist thermal bubble.

\begin{tabular}{l|lllll}
\hline \hline No. & start position & end position & $\delta_{x}[\mathrm{~m}]$ & $\delta_{t}[\mathrm{~s}]$ & $\tau$ \\
\hline 0 & $(0,0,0)$ & $(3600,3600,2400)$ & 50.0 & 1.44 & 0.5017 \\
\hline 1 & $(1000,1000,500)$ & $(2600,2600,2200)$ & 25.0 & 0.72 & 0.5034 \\
\hline 2 & $(1200,1200,700)$ & $(2400,2400,1800)$ & 12.5 & 0.36 & 0.5069 \\
\hline 3 & $(1400,1400,800)$ & $(2200,2200,1600)$ & 6.25 & 0.18 & 0.5138 \\
\hline \hline
\end{tabular}

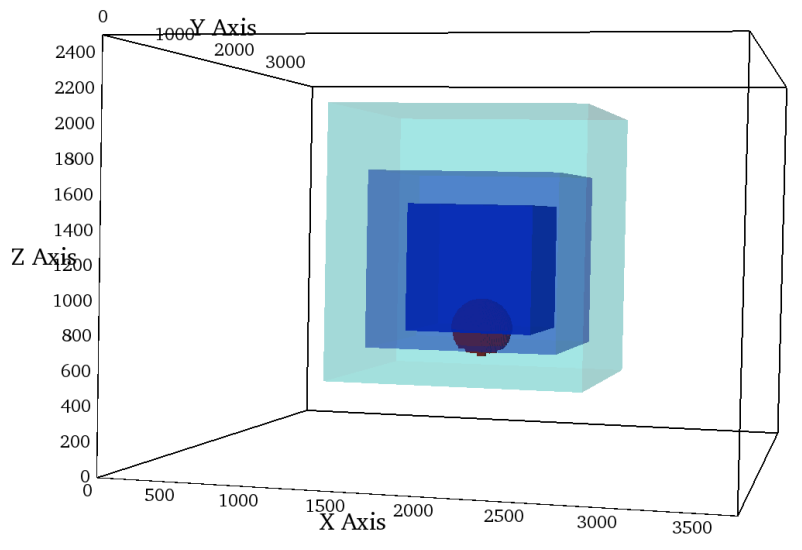

FIG. 10. Setup of three-dimensional geometry and subdomains for simulation of $3 \mathrm{D}$ rising moist thermal bubble.

The present lattice Boltzmann method with condensation model (2eq) incorporated with grid refinement technique are assessed in this case. The sub-domains with different grid resolution is used as illustrated in Table III and Fig. 10. The kinematic viscosity $\nu$ is assigned $1 \mathrm{~m}^{2}$ $\mathrm{s}^{-1}$ and Prandtl number $\operatorname{Pr}_{\theta}$ as well as $\operatorname{Pr}_{q}$ are set to unity in the simulations. The time step is calculated with a sound speed in physical unit $c_{s p}=20 \mathrm{~m} \mathrm{~s}^{-1}$. The corresponding non-dimensional relaxation time parameters are also given in Tab. III.

Results of the simulations with grid refinement after 2, 4 and 6 min are presented in Fig. 11 and Fig. 12, respectively. The results in the central sub-domain [1450, $2150] \times[600,1800] \mathrm{m}^{2}$ in $\mathrm{x}$-plane are displayed. The results obtained by the present LB method with the 2eq condensation model are in excellent agreement with the
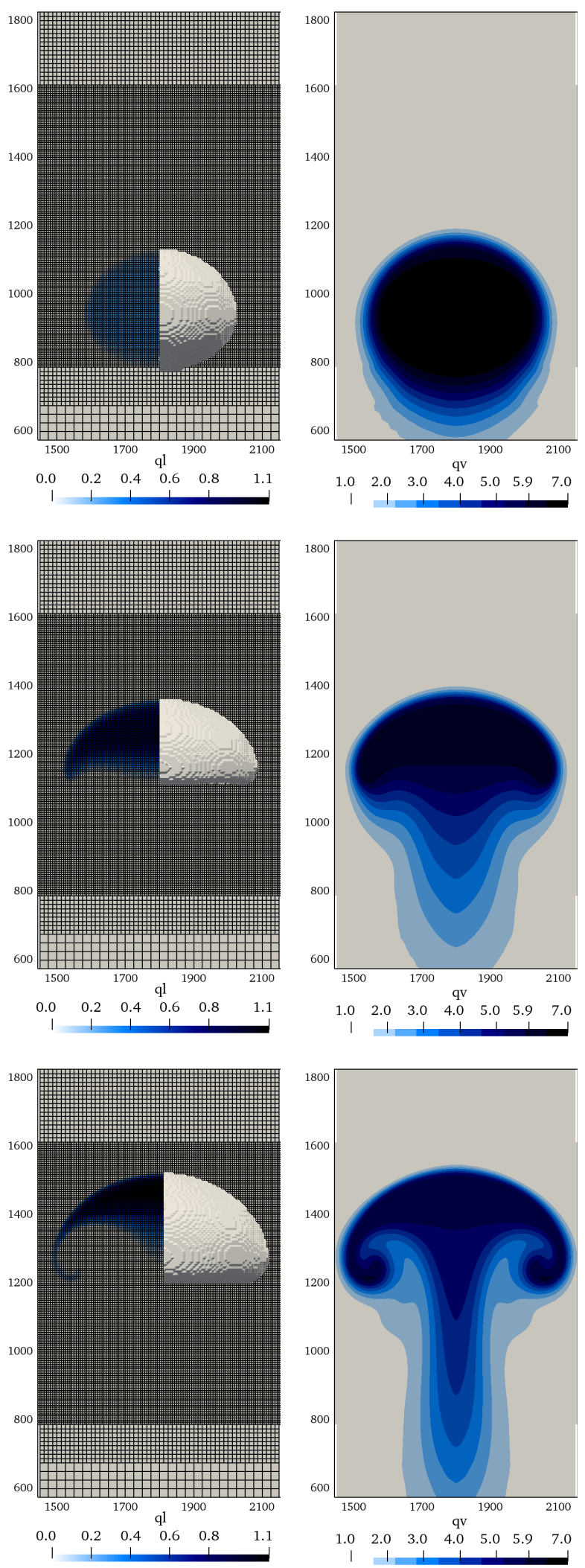

FIG. 11. Contours of liquid humidity field (left) and vapor humidity field (right) at 2, 4 and 6 min (top to bottom)as obtained by the present LB method with 2eq. condensation model. 
results in Ref. [see 59, Fig.1].

In order to quantitatively validate the proposed LB model, the liquid humidity and vertical velocity along mid-line are plotted in Fig. 13. The computed velocities at 2, 4 and 6 min are confirmed closely with benchmark solution in Ref. [59]. The benchmark solution was obtained by an anelastic solver using variables $\left(\theta, q_{v}, q_{l}\right)$ on minimum grid spacing of $6.25 \mathrm{~m}$.

\section{Shallow Cumulus Convection}

At last, a shallow cumulus convection is simulated to evaluate the capability of the present LB method to capture the moist thermodynamics and its interaction with the meteorological flows. The shallow cumulus convection simulations follows the setup of the Barbados Oceanographic and Meteorological Experiment (BOMEX) model inter-comparison case [35]. This is the most prevalent shallow cumulus large-eddy simulation (LES) case.

In this case, an altitude-dependent geostrophic wind $U_{g}$ is given by a linear formula $U_{g}=\left(-10+1.8 \times 10^{-3} z\right)$ $\mathrm{m} \mathrm{s}^{-1}$ and the Coriolis parameter is set to $f=0.376 \times$ $10^{-4} \mathrm{~S}^{-1}$. The initial conditions for velocity, liquid water potential temperature and total water mixing ratio are linear profiles following the values given on Table IV. The temperature and humidity surface fluxes are $8 \times 10^{-3}$ $\mathrm{K} \mathrm{m} \mathrm{s}^{-1}$ and $5.2 \times 10^{-5} \mathrm{~m} \mathrm{~s}^{-1}$, respectively. The shear stresses are prescribed by $\overline{u_{i} w}=-u_{*}^{2} u_{i} /\left(u_{1}^{2}+u_{2}^{2}\right)^{1 / 2}$, with $u_{*}=0.28 \mathrm{~m} \mathrm{~s}^{-1}$.

TABLE IV. Initial conditions for shallow cumulus convection.

\begin{tabular}{rccll}
\hline \hline Height $(\mathrm{m})$ & $q_{t}\left(\mathrm{~g} \mathrm{~kg}^{-1}\right)$ & $\theta_{l}(\mathrm{~K})$ & $u\left(\mathrm{~m} \mathrm{~s}^{-1}\right)$ & $v\left(\mathrm{~m} \mathrm{~s}^{-1}\right)$ \\
\hline 0 & 17.0 & 298.7 & -8.75 & 0 \\
520 & 16.3 & 298.7 & & \\
700 & & & -8.75 & \\
1480 & 10.7 & 302.4 & & \\
2000 & 4.2 & 308.2 & & 0 \\
3000 & 3.0 & 311.85 & -4.61 & 0 \\
\hline \hline
\end{tabular}

Moreover, additional terms are added to represent the large-scale forcing which could not be represented directly in the LES. The source terms of momentum conservation equations, temperature equation and water equations are parameterized considering the effects of largescale subsidence, radiative cooling and moisture effects. The details on large-scale forcing were reported in [35].

The computational domain size is $5000 \mathrm{~m} \times 5000 \mathrm{~m} \times$ $3000 \mathrm{~m}$. Here, the lattice Boltzmann method with condensation scheme $2 \mathrm{eq}$ is used to compute that case considering on grid resolution of $\delta_{x}=40 \mathrm{~m}$. The time step is equal to $0.27 \mathrm{~s}$ with $c_{s p}$ being $85 \mathrm{~m} \mathrm{~s}^{-1}$ in the simulation. The kinematic viscosity of air is $1.5 \times 10^{-5}$ $\mathrm{m}^{2} \mathrm{~s}^{-1}$ and Prandtl number $\operatorname{Pr}_{\theta}=\operatorname{Pr}_{q}=0.71$ for potential temperature and water fraction are used in the simulation. Thus the non-dimensional relaxation time $\tau$
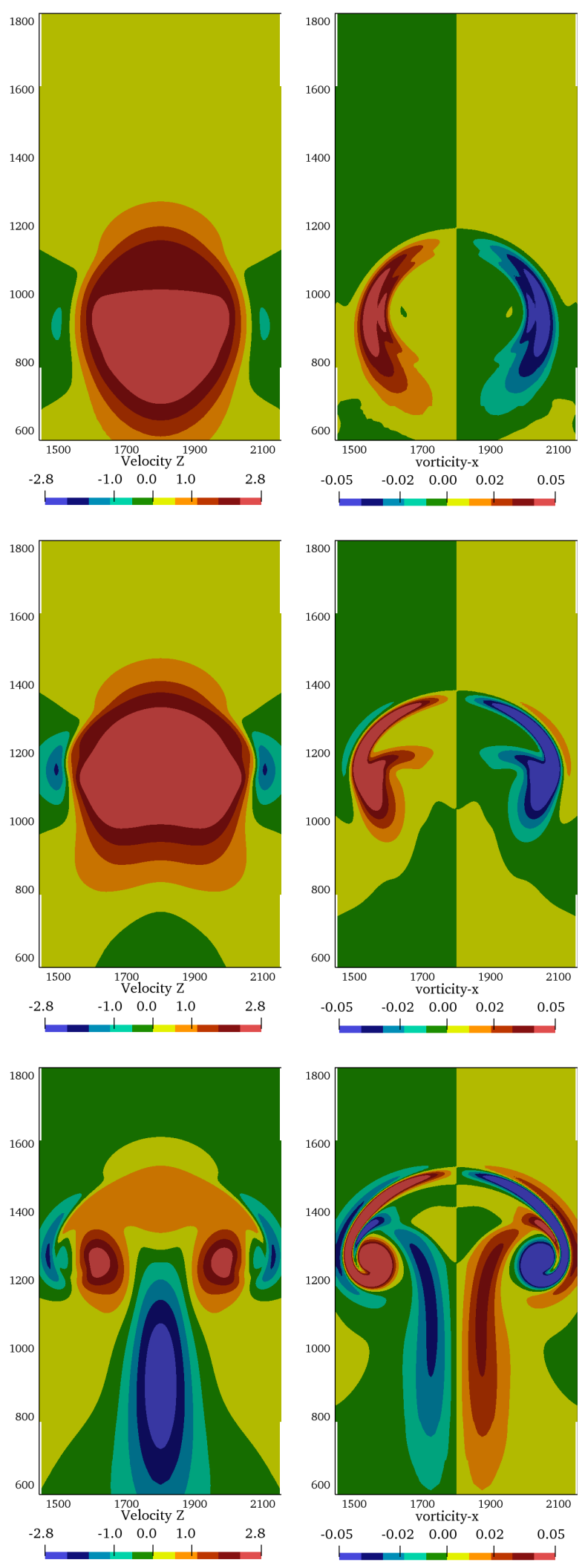

FIG. 12. Contours of vertical velocity (left) and x component of vorticity (right) at 2,4 and $6 \mathrm{~min}$ (top to bottom) as obtained by the present LB method with 2 eq model and grid refinement. 

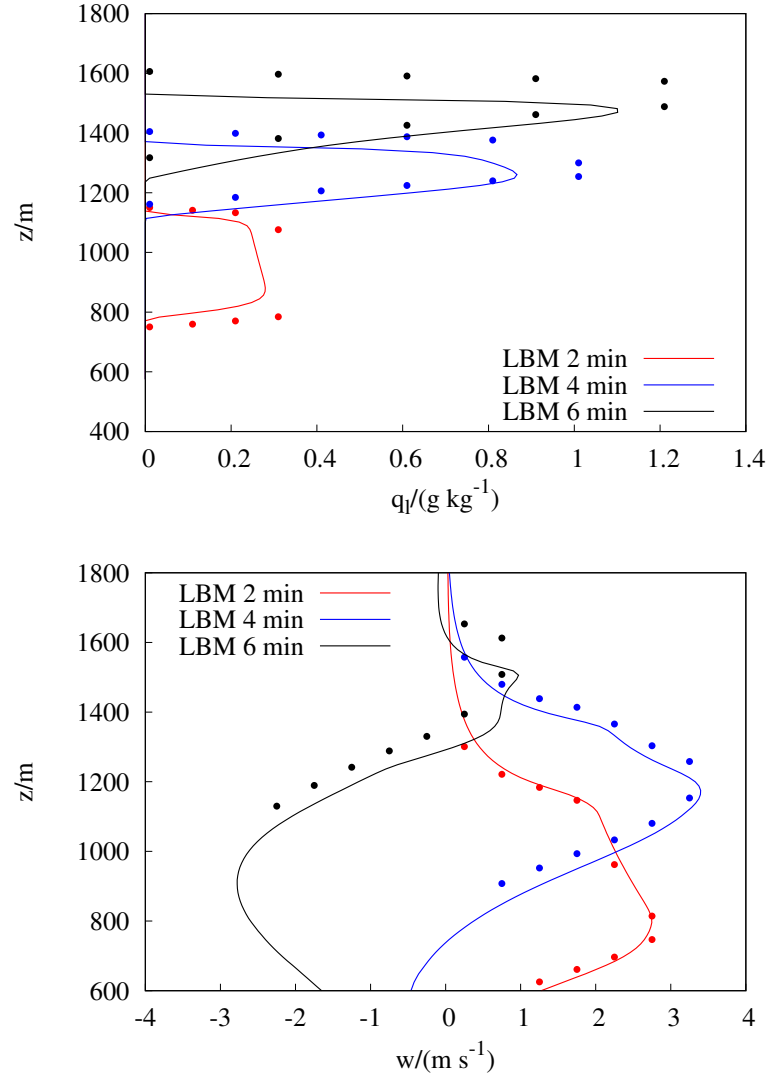

FIG. 13. Profile of liquid humidity and vertical velocity along vertical middle line at 2, 4, $6 \mathrm{~min}$. The symbols represent benchmark solution in Ref. [59].

based on the kinematic viscosity is approximately equal to $\left(0.5+7 \times 10^{-9}\right)$. The classical Smagorinsky model is adopted as the subgrid model in the large-eddy simulation [60] and the surface model of the horizontal momentum components, temperature and humidity is using the Monin - Obukhov similarity theory (MOST) [61]. The incorporating LES within framework of lattice Boltzmann method for atmospheric boundary layer flows are detailed in [62]. The constant of the Smagorinsky subgrid viscosity model is taken equal to $C_{S}=0.15$ and turbulent Prandtl numbers of $K_{t}=0.33$ and $K_{q}=0.33$ are adopted both for potential temperature and water fraction equations. The simulation is carried out over 6 hour of physical time. The statistics are computed averaging the solution over 1 hour.

Figure 14 shows the computed vertical mean profiles of velocities, potential temperature, vapor water and liquid water. All of the results are in good agreement with the reference data from [35], proving the accuracy of the proposed LB model with condensation scheme. The mixed region below the altitude of $540 \mathrm{~m}$ is well captured, demonstrating the accuracy of the wall model with complex thermophysics and cloud dynamics. Furthermore, the conditionally unstable layer from $540 \mathrm{~m}$ to $1500 \mathrm{~m}$,
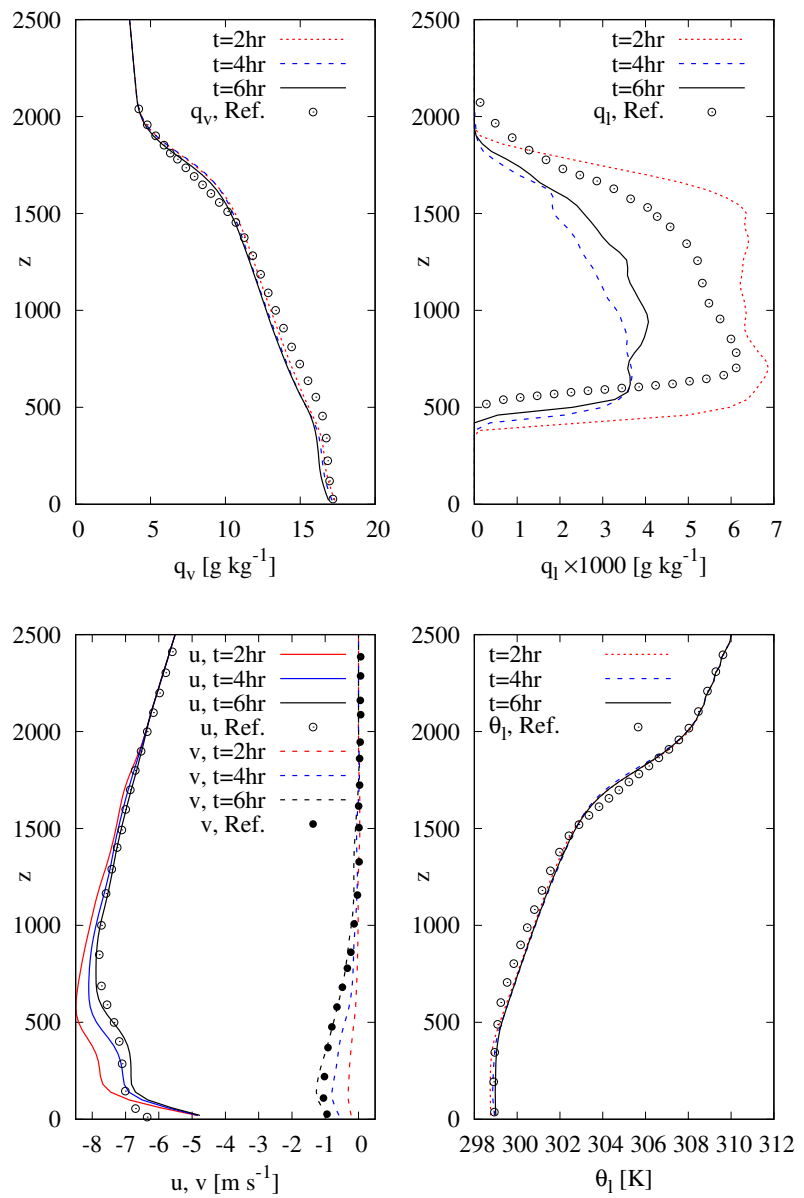

FIG. 14. Mean profiles of vapor water (top, left), liquid water (top, right), velocity (bottom, left) and potential temperature (bottom, right) obtained in the convective cumulus convection case. The reference data in symbols are results at $6 \mathrm{hr}$ from [35].

and the inversion layer from $1500 \mathrm{~m}$ to $2000 \mathrm{~m}$ are also clearly observed in the results.

Figure 15 shows iso-surfaces of liquid water and temperature in the full computational domain. In order to emphasize interaction between temperature field and humidity field as well as instantaneous flow features of LES, cloud (iso-surface of liquid water) is displayed. The results is obtained at time of the 1.5th hour. To highlight the thermal plumes near surface, the potential temperature is chosen equal to $298.7 \mathrm{~K}$, and the color represents the altitude. The results are also at time of the 1.5 th hour with $\delta_{x}=40 \mathrm{~m}$ in the figure. The thermal plumes are well generated by the coupling between LB core and passive scalars solvers. It can be observed that the instantaneous formation of cloud is well captured by the present lattice Boltzmann model with condensation scheme. 


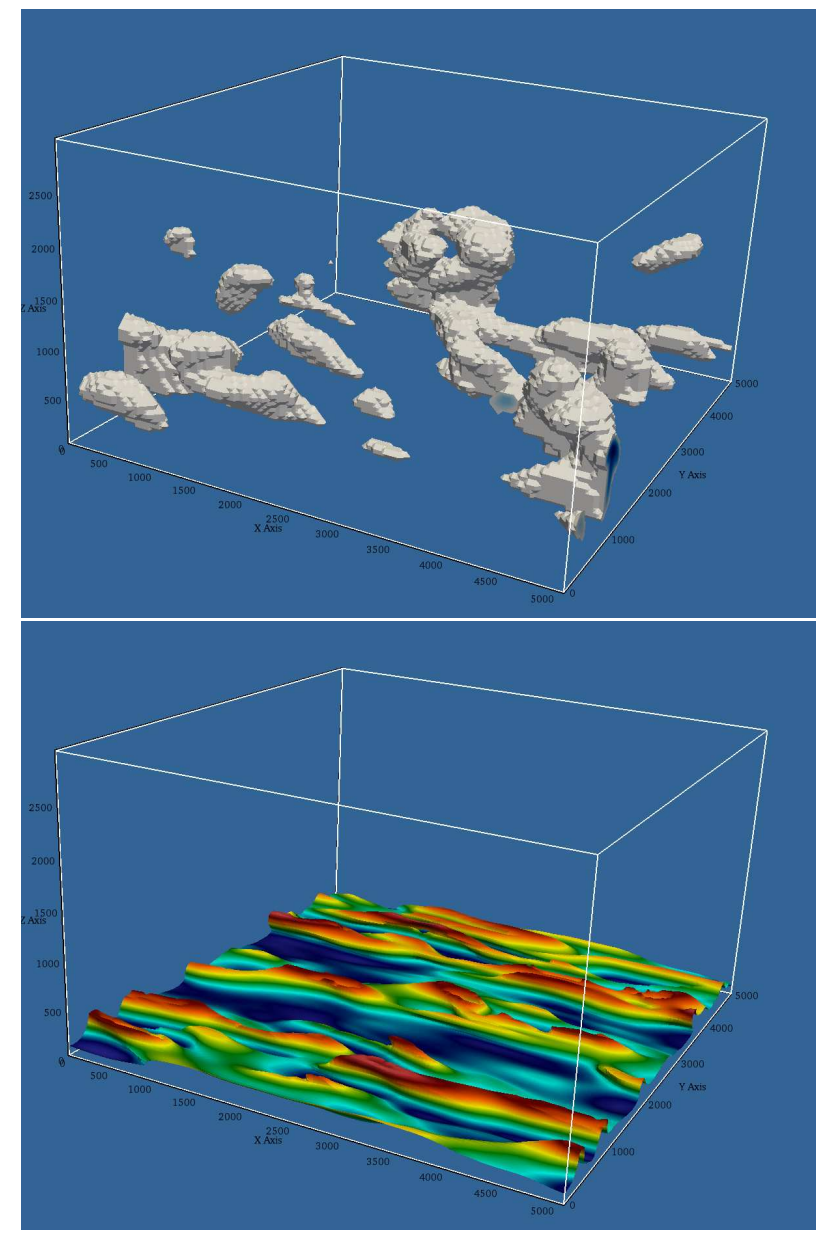

FIG. 15. Iso-surface of liquid water (top) and temperature (bottom) at the 1.5 hour for shallow cumulus convection.

\section{CONCLUSION}

In this paper, a hybrid LB based condensation models for moist atmospheric convection is proposed. A recursive regularized BGK model with forcing term is employed in LB equation and the finite volume scheme is adopted on temperature and water transport equations. Four benchmark problem have been used to assess the LB method incorporated two condensation models. Both the 1eq model and the 2eq model present accurate solution in a 2D moist rising bubble simulation. In summary, the 2eq model is more general in both shallow and deep atmospheric flows and the 2eq model is preferred as a framework for involving the precipitation microphysics in the future. The proposed LB approach for humid air and cloud dynamics has been assessed considering a 3D moist rising bubble simulation and cloudy convective atmospheric boundary layer with phase change and large scale sources. To further develop a LB model for moist atmospheric deep convection, an appropriate model under the anelastic approximation will be proposed in the future.

\section{ACKNOWLEDGEMENTS}

The first author would like to gratefully acknowledge to Dr. Johann Miranda for fruitful discussions. This work was supported by the French project CLIMB, with the financial support of BPIFrance (Grant P3543-24000), in the framework of the program "Investissement d'Avenir: Calcul Intensif et Simulation Numérique". This work was performed using HPC resources from GENCITGCC/CINES (Grant 2018-A0052A07679). This work was also supported by project of ProLB software (http://www.prolb-cfd.com).
[1] B. Stevens, Annu. Rev. Earth Planet. Sci. 33, 605 (2005).

[2] L. Mortensen, M. Woloszyn, C. Rode, and R. Peuhkuri, Journal of Building Physics 30, 279 (2007).

[3] M. Zerroukat and T. Allen, Journal of Computational Physics 290, 55 (2015).

[4] W. P. O'Neill and R. Klein, Atmospheric Research 142, 133 (2014).

[5] P. R. Bannon, Journal of the Atmospheric Sciences 59, 1967 (2002).

[6] W. W. Grabowski and P. K. Smolarkiewicz, Monthly Weather Review 118, 2082 (1990).

[7] J. W. M. Cuijpers and P. G. Duynkerke, "Large eddy simulation of trade wind cumulus clouds," (1993).

[8] M. Duarte, A. S. Almgren, K. Balakrishnan, J. B. Bell, and D. M. Romps, Monthly Weather Review 142, 4269 (2014).

[9] P. K. Smolarkiewicz and L. G. Margolin, Journal of Computational Physics 140, 459 (1998).

[10] J. Jacob and P. Sagaut, Building and Environment 139, 110 (2018).

[11] S. Wilhelm, J. Jacob, and P. Sagaut, Physics of Fluids 30, 065111 (2018).
[12] L.-S. Luo, M. Krafczyk, and W. Shyy, Encyclopedia of Aerospace Engineering (2010).

[13] Y. Feng, M. Tayyab, and P. Boivin, Combustion and Flame 196, 249 (2018).

[14] G. Feingold, I. Koren, H. Wang, H. Xue, and W. A. Brewer, Nature 466, 849 (2010).

[15] R. Geist, J. E. Steele, and J. Westall, in NPH (2007) pp. 23-30.

[16] Q. Li, K. Luo, Q. Kang, Y. He, Q. Chen, and Q. Liu, Progress in Energy and Combustion Science 52, 62 (2016).

[17] P. J. Dellar, Physical Review E - Statistical, Nonlinear, and Soft Matter Physics 65, 1 (2002).

[18] M. Waruszewski, C. Kühnlein, H. Pawlowska, and P. K. Smolarkiewicz, Journal of Computational Physics 359, 361 (2018).

[19] L.-S. Luo, W. Liao, X. Chen, Y. Peng, W. Zhang, et al., Physical Review E 83, 056710 (2011).

[20] P. L. Bhatnagar, E. P. Gross, and M. Krook, Phys. Rev. 94, 511 (1954).

[21] P. Lallemand and L. S. Luo, Physical Review E 68, 036706 (2003). 
[22] G. Di Ilio, B. Dorschner, G. Bella, S. Succi, and I. Karlin, Journal of Fluid Mechanics 849, 35 (2018).

[23] N. I. Prasianakis, S. S. Chikatamarla, I. V. Karlin, S. Ansumali, and K. Boulouchos, Mathematics and Computers in Simulation 72, 179 (2006).

[24] N. Frapolli, S. Chikatamarla, and I. Karlin, Computers \& Fluids (2018).

[25] J. Latt and B. Chopard, Mathematics and Computers in Simulation 72, 165 (2006).

[26] K. K. Mattila, P. C. Philippi, and L. A. Hegele Jr, Physics of Fluids 29, 046103 (2017).

[27] M. Geier, M. Schönherr, A. Pasquali, and M. Krafczyk, Computers \& Mathematics with Applications 70, 507 (2015).

[28] F. Hajabdollahi and K. N. Premnath, Physical Review E 97, 063303 (2018).

[29] R. K. Freitas, A. Henze, M. Meinke, and W. Schröder, Computers \& Fluids 47, 115 (2011).

[30] M. Gehrke, C. Janßen, and T. Rung, Computers \& Fluids 156, 247 (2017).

[31] I. V. Karlin, F. Bösch, and S. Chikatamarla, Physical Review E 90, 031302 (2014).

[32] L. Hegele Jr, A. Scagliarini, M. Sbragaglia, K. Mattila, P. Philippi, D. Puleri, J. Gounley, and A. Randles, Physical Review E 98, 043302 (2018).

[33] C. Zhuo and P. Sagaut, Physical Review E 95, 063301 (2017).

[34] J. Jacob, O. Malaspinas, and P. Sagaut, Journal of Turbulence, 1 (2018).

[35] A. P. Siebesma, C. S. Bretherton, A. Brown, A. Chlond, J. Cuxart, P. G. Duynkerke, H. Jiang, M. Khairoutdinov, D. Lewellen, C.-H. Moeng, E. Sanchez, B. Stevens, and D. E. Stevens, J. Atmos. Sci. 60, 1201 (2003).

[36] J. W. Deardorff, Journal of Applied Meteorology 15, 98 (1976).

[37] P. Bougeault, Journal of the Atmospheric Sciences 38, 2414 (1981).

[38] G. Sommeria, Journal of the Atmospheric Sciences 33, 216 (1976).

[39] S. Chen and G. D. Doolen, Annual Review of Fluid Mechanics 30, 329 (1998).

[40] Y. Qian, D. D'Humières, and P. Lallemand, Europhysics Letters 17, 479 (1992).

[41] X. He, S. Chen, and G. D. Doolen, Journal of Computational Physics 146, 282 (1998).

[42] T. Krüger, H. Kusumaatmaja, A. Kuzmin, O. Shardt, G. Silva, and E. M. Viggen, The Lattice Boltzmann Method: Principles and Practice (Springer, 2016).

[43] P. Dellar, Comput. Mathematics Applications 65, 129 (2013).

[44] Z. Guo, C. Zheng, and B. Shi, Physical Review E 65, 046308 (2002).

[45] D. Siebert, L. Hegele Jr, and P. Philippi, Physical Review E 77, 026707 (2008).

[46] P. J. Dellar, Journal of Computational Physics 259, 270 (2014).

[47] O. Malaspinas, arXiv preprint arXiv:1505.06900 (2015).

[48] G. Wissocq, P. Sagaut, and J.-F. Boussuge, Journal of Computational Physics 380, 311 (2019).

[49] Y. Feng, P. Boivin, J. Jacob, and P. Sagaut, Journal of Computational Physics 394, 82 (2019).

[50] X. Shan, X.-F. Yuan, and H. Chen, Journal of Fluid Mechanics 550, 413 (2006).

[51] X. Shan, Physical Review E 55, 2780 (1997).
[52] Y.-L. Feng, S.-L. Guo, W.-Q. Tao, and P. Sagaut, International Journal of Heat and Mass Transfer 125, 1379 (2018).

[53] K. H. Kim, C. Kim, and O.-H. Rho, Journal of Computational Physics 174, 38 (2001).

[54] C. Hirsch, Numerical computation of internal and external flows: The fundamentals of computational fluid dynamics (Elsevier, 2007).

[55] J. Latt, B. Chopard, O. Malaspinas, M. Deville, and A. Michler, Physical Review E 77, 056703 (2008).

[56] "ProLB: high-fidelity CFD in exceptional turnaround times," http://www prolb-cfd.com (2019).

[57] N. Ouertatani, N. B. Cheikh, B. B. Beya, and T. Lili, Comptes Rendus Mecanique 336, 464 (2008).

[58] W. W. Grabowski and T. L. Clark, Journal of the Atmospheric Sciences 48, 527 (1991).

[59] W. W. Grabowski and T. L. Clark, Journal of the Atmospheric Sciences 50, 555 (1993).

[60] P. Sagaut, Computers \& Mathematics with Applications 59, 2194 (2010).

[61] A. S. Monin and A. M. Obukhov, Contrib. Geophys. Inst. Acad. Sci. USSR 151, e187 (1954).

[62] Y. Feng, J. Miranda, J. Jacob, and P. Sagaut, Journal of Advances in Modeling Earth Systems, In preparation (2019). 